\title{
desenvolvimento QUESTÂOO
}

\section{LA CARTERA CREDITICIA DE LOS AGRICULTORES COLOMBIANOS: Un caso de Estudio para una Entidad Financiera del Sector Real}

\author{
http://dx.doi.org/10.21527/2237-6453.2021.57.11923
}

Recebido em: $12 / 2 / 2021$

Aceito em: 28/6/2021

\author{
Santiago Moreno López ${ }^{1}$, Nadja Simone Menezes Nery de Oliveira², \\ Jandir Ferrera de Lima $^{3}$
}

\begin{abstract}
RESUMEN
El presente trabajo pretende realizar un diagnóstico sobre la problemática del acceso a la financiación y a través de un caso de estudio demostrar que los agricultores colombianos tienen un buen hábito de pago en sus obligaciones financieras. Con este fin, y con base en una muestra con un total de 43.295 desembolsos entre los años 2018-2019 se identificarán las zonas geográficas del país más riesgosas de acuerdo con el incumplimiento de los clientes en sus obligaciones financieras, y se analizó la actividad principal de los departamentos colombianos para tratar de validar la correlación con la voluntad de pago de los agricultores. Los resultados mostraron que, con relación al tiempo de ocupación, los perfiles menos atractivos para incentivar el acceso al crédito son los prestadores de servicio o contratistas y los agricultores dadas las tasas de impago, $20 \%$ y $18 \%$ respectivamente. Con relación al ranking de impago por departamentos se destaca que aquellos donde la ganadería ocupa un lugar representativo en su actividad económica presentan las tasas más altas de impago respecto a departamentos donde predomina la agricultura.
\end{abstract}

Palabras-clave: Inclusión financiera; agricultores; sector rural; impago crediticio.

\section{A CARTEIRA DE CRÉDITO DOS AGRICULTORES COLOMBIANOS:} UM ESTUDO DE CASO PARA UMA ENTIDADE FINANCEIRA DO SETOR REAL

\section{RESUMO}

Este artigo tem por objetivo realizar um diagnóstico sobre o problema do acesso ao financiamento e, através de um estudo de caso, demonstrar que os agricultores colombianos têm um bom hábito de pagar suas obrigações financeiras. Para esse fim, e com base em uma amostra com um total de 43.295 desembolsos entre os anos de 2018 e 2019 , foram identificadas as áreas geográficas do país mais arriscadas de acordo com o descumprimento de suas obrigações financeiras por parte dos clientes, também serão analisadas a atividade principal dos estados colombianos para tentar validar a correlação com a disposição dos agricultores a pagar. Os resultados mostraram que, em relação ao tempo de ocupação, os perfis menos atrativos para incentivar o acesso ao crédito são prestadores de serviços ou contratados e os agricultores, considerando as taxas de inadimplência de $20 \%$ e $18 \%$, respectivamente. No que diz respeito à classificação de não pagamento por departamentos, vale ressaltar que aqueles onde o gado ocupa um lugar representativo em sua atividade econômica têm as mais altas taxas de inadimplência em relação aos departamentos onde a agricultura predomina.

Palavras-chave: Inclusão financeira; agricultores; setor rural; padrão de crédito.

\footnotetext{
${ }^{1}$ Universidad del Valle. Cali, Valle del Cauca, Colombia.

${ }^{2}$ Autora correspondente. Universidad del Valle. Calle 100 N 00-00 Edif. 387 Piso 4. Cali, Valle del Cauca, Colombia. Codigo Postal 76001. http://lattes.cnpq.br/230355783866312. https://orcid.org/0000-0001-5374-7261.nadja.menezes@correounivalle.edu.co

${ }^{3}$ Universidade Estadual do Oeste do Paraná - Unioeste. Toledo/PR, Brasil.
} 


\section{INTRODUCCIÓN}

El sistema financiero colombiano ha cumplido un rol fundamental en el crecimiento del entorno económico del país al impulsar a través del apalancamiento monetario el desarrollo de proyectos y programas de personas y empresas, generando una disminución de la pobreza e incentivando la creación y el fortalecimiento de la base empresarial. La inclusión financiera ha permitido a las comunidades un mayor acceso a la bancarización, lo que les permite financiar la adquisición de bienes o servicios para mejorar su calidad de vida, hecho que se refleja en el índice de bancarización, que evidencia un avance importante al pasar del 73,9\% en el 2014 al $81,4 \%$ en el 2018. El indicador de bancarización representa el porcentaje de adultos que tienen acceso, al menos, a un producto financiero, ya sea de ahorro o crédito formal, en un establecimiento de crédito, ONG o cooperativa de ahorro (ASOBANCARIA, 2018).

El incumplimiento en el pago de los créditos por parte de los clientes representa un riesgo para la actividad de las instituciones financieras, pues genera un problema de pérdida en los activos obligándolas a elevar el costo de los productos o adoptar medidas para reducir el acceso a la financiación de comunidades vulnerables. Por lo que es importante para estas instituciones, definir e implementar metodologías que permitan evaluar la viabilidad del retorno de inversión en las colocaciones de crédito y mejorar la toma de decisiones que maximicen la rentabilidad.

Es importante tener en cuenta dentro de la estimación del riesgo crediticio la influencia del mercado laboral colombiano ya que presenta una heterogeneidad de situaciones en la calidad del empleo donde se destaca la informalidad $(47,0 \%)$, el desempleo $(10,2 \%)$ y los bajos ingresos $(57,3 \%)^{4}$; además de las amplias diferencias que se presentan en la calidad de vida entre el sector urbano y el rural, especialmente en ámbitos como salud, educación, servicios públicos y acceso a la financiación (DANE, 2019a,b; FEDESARROLLO, 2018).

En 2018, el porcentaje de personas clasificadas como pobres ${ }^{5}$ en Colombia respecto al total de la población fue $27,0 \%$ y como pobres extremos fue del 7,2\%. En las cabeceras la proporción de pobres fue $24,4 \%$ y de pobres extremos fue del $4,9 \%$. En los centros poblados y rural disperso ${ }^{6}$ fue el porcentaje de personas clasificadas como pobres fue del $36,1 \%$ y como pobres extremos fue $15,4 \%$, lo que significa que la incidencia de la pobreza y de la pobreza extrema en los centros poblados y rural disperso equivale a 1,5 y 3,1 veces la incidencia en las cabeceras, respectivamente (DANE, 2018).

Estas condiciones macroeconómicas limitan y disuaden a la mayoría de las entidades financieras formalmente constituidas en el país a expandir sus productos y servicios a las zonas rurales, pues no representan un nicho de mercado atractivo que garantice el retorno de la inversión. Sin embargo, el presente trabajo pretende realizar un diagnóstico sobre la problemática

\footnotetext{
4 "...el salario mínimo representa el $57,3 \%$ del promedio de los salarios de las personas ocupadas en el país...". (FEDESARROLLO, 2018).

${ }^{5}$ En esta medida se considera la pobreza monetaria. Esta cifra se construye a partir de la medición del ingreso per cápita corriente de la unidad del gasto (UG), o sea, el ingreso corriente de la unidad de gasto dividido por el total de integrantes de esta; y se compara con el costo monetario de adquirir una canasta de alimentos (DANE, 2018).

${ }^{6}$ La categoría rural disperso se refiere a aquellos municipios y Áreas No Municipalizadas -AMN- que tienen cabeceras pequeñas y densidad poblacional baja, o sea, menos de $50 \mathrm{hab} . / \mathrm{KM}^{2}$, al paso que la categoría rural corresponde a los municipios que tienen cabeceras de menor tamaño (menos de 25 mil habitantes) y presentan densidades poblacionales intermedias, entre $10 \mathrm{hab} . / K M^{2}$ y 100 hab./KM ${ }^{2}$ (DNP, 2014).
} 
del acceso a la financiación y a través de un caso de estudio demostrar que los agricultores colombianos tienen un buen hábito de pago de sus obligaciones financieras; por lo que es indispensable promover políticas públicas e incentivos a las organizaciones privadas para que integren con prioridad a las comunidades vulnerables al sector financiero y contribuir al Programa de las Naciones Unidas para el Desarrollo (PNUD), que tiene como uno de sus pilares la erradicación de la pobreza usando la financiación como un medio.

Este estudio cobra importancia al focalizar el análisis de riesgo crediticio por perfil de cliente y promover que las entidades financieras involucren a las comunidades vulnerables, generando una mayor rentabilidad producto de su actividad y un impacto social al brindar acceso a créditos con mejores beneficios en cuanto a tasa de interés y plazo, respecto a vías informales de apalancamiento como los prestamistas gota a gota ${ }^{7}$; buscando promover una sociedad más equitativa en oportunidades y lograr alcanzar un mayor crecimiento económico.

Igualmente, la elección del tema se justifica ya que se entiende que la inclusión financiera a través del crédito es importante como instrumento para el desarrollo de cualquier país pues ella contribuye a que individuos de la parte baja de la base piramidal de una sociedad puedan superar la pobreza. Con respecto a las áreas rurales, el crédito permite mantener y ampliar los estándares de productividad agrícola, además de incrementar el acceso a insumos y equipos agrícolas.

El artículo está dividido en tres sesiones. En la primera, se realiza una breve reseña bibliográfica en la que se contextualiza y se describe la problemática del acceso a la financiación principalmente en el sector rural. Sigue, el análisis empírico y los resultados encontrados sobre las tasas de impago reportadas de acuerdo con diferentes perfiles de clientes y se analiza el desempeño del hábito de pago de los agricultores. Finalmente, se presentan las conclusiones.

\section{REVISIÓN DE LA LITERATURA}

El desarrollo económico es un cambio desequilibrado, que crea una nueva condición económica, basada en tres factores: innovaciones, crédito bancario y la acción del emprendedor innovador, ya sea un emprendedor rural o urbano (SCHUMPETER, 1982).

Para producir e introducir innovaciones en el sistema económico, es necesario obtener crédito para la adquisición de bienes y servicios. El crédito es fundamental para el proceso económico, ya que proporcionar crédito a través de la capacidad del sistema financiero para crear poder adquisitivo, a través de su apalancamiento, es el motor del proceso de cambio que caracteriza el desarrollo económico. La comercialización del crédito debe tener el propósito de financiar el desarrollo (SCHUMPETER, 1982).

Hay dos tipos de crédito: crédito normal, es decir, crédito que crea el derecho al consumo, demandando bienes y servicios existentes; crédito para el desarrollo, que también otorga el derecho al consumo, pero es un insumo para la producción y la creación de empleo. El crédito para el desarrollo, es decir, para el emprendedor que hará nuevas combinaciones en sus actividades, tiene su característica principal en el hecho de que utiliza recursos financieros que estaban inactivos (SCHUMPETER, 1982).

\footnotetext{
Prestamista informal.
} 
Los factores que afectan la cantidad de capital inactivo comienzan a determinar la expansión o contracción de las concesiones crediticias y el ritmo de crecimiento y desarrollo económico. El crédito que convierte cualquier tipo de recurso financiero inactivo en capital monetario puede llamarse crédito de inversión (o crédito de capital), ya que este tipo de crédito siempre es una transferencia de dinero mediante la adquisición de bienes y servicios (HILFERDING, 1985).

El crédito de inversión expande la función del capital productivo, a través de la circulación del capital monetario en sí mismo, ya que convierte el capital monetario inactivo en capital activo. También implica la transferencia de una suma de dinero que alguien no podrá invertir en capital a otra persona, que asignará ese recurso como inversión, conservando así el valor del capital y permitiendo que circule nuevamente en un período de tiempo futuro. La concesión de crédito busca suprimir la ociosidad del capital monetario (HILFERDING, 1985).

El desempeño de las instituciones financieras es inicialmente un mediador de los medios de pago en circulación. Después de esta etapa, la institución financiera convierte los recursos monetarios inactivos en activos, ya que transfiere a los empresarios innovadores las cantidades capturadas y concentradas anteriormente. Para obtener recursos financieros y ponerlos a disposición de los empresarios, las instituciones financieras deben fomentar la inclusión financiera.

\section{La Inclusión Financiera}

En la actualidad existen diversas interpretaciones sobre la definición y el alcance de la inclusión financiera, pero se puede entender como el proceso de integración de los servicios financieros formales (ahorro, créditos y seguros) a las actividades económicas cotidianas de los individuos, que pueden contribuir al crecimiento económico en la medida en que se logren reducir los costos de financiación y transacción, y adicionalmente se ofrezca un manejo seguro y eficiente de los recursos, tanto para los hogares como para las empresas.

En ese contexto, la inclusión financiera reside en la promoción de un acceso oportuno, eficiente y apropiado a un grado de productos y servicios financieros regulados, igualmente como la expansión de su utilización por parte de todos los fragmentos de la sociedad a través de la implementación de acciones personificadas y transformadoras que contengan educación y conocimientos financieros con el objetivo de suscitar el bienestar, y la inserción económica y social (OCDE/INFE, 2012).

Se pueden mencionar varios beneficios de la inclusión financiera en general; pero el simple acceso a los productos financieros no implica un impacto positivo en sí, sino que es el uso efectivo de estos productos el vehículo para suavizar el ingreso y los ciclos de consumo. Por ejemplo, con los productos de ahorro y crédito, los hogares tienen la posibilidad de distribuir su riqueza de forma intertemporal y disfrutar de sus rentas a lo largo de su ciclo de vida (MORDU$\mathrm{CH}, 1995)$.

Hay tres tipos de beneficios del microcrédito: primero de carácter social, al aumentar los ingresos que mejoran la calidad de vida de los hogares. Segundo, sobre el medio microempresarial, porque a este nicho están especialmente dirigidos, al fomentar nuevas microempresas o para mejorar las que ya existen. Y finalmente, el impacto en los mercados financieros porque aumenta la oferta de productos y servicios financieros y expande el mercado de las entidades financieras generando una mayor rentabilidad (RODRÍGUEZ GARCÉS, 2008). 
El microcrédito se combina con los siete principios del cooperativismo, mencionados a continuación: i) las cooperativas son entidades abiertas a cualquier persona; ii) las cooperativas son organizaciones democráticas, en las que sus miembros participan en la gestión; iii) el aporte equitativo al capital de la cooperativa, con posibilidad de remunerar ese capital; iv) las cooperativas son organizaciones autónomas de autoayuda, controladas por sus miembros; v) son responsables de promover la educación y la formación de los miembros; vi) las cooperativas pueden trabajar juntas, mediante la intercooperación; vii) las cooperativas trabajan por el desarrollo sostenible de las comunidades en las que operan (DAMBROS; FERRERA DE LIMA; FIGUEIREDO, 2009). Dentro de los siete principios, el microcrédito fortalece las condiciones de vida de los cooperativistas y también se convierte en un instrumento para la autosostenibilidad de la cooperativa. Al mejorar las condiciones de su negocio y el capital de trabajo, el cooperativista podrá destinar más excedentes al desarrollo de la cooperativa, generar reservas y obtener mayores excedentes en proporción a sus transacciones. Por un lado, el microcrédito fortalece los indicadores de ingresos y producción y, por otro, fomenta la inclusión financiera.

La inclusión financiera trasciende los indicadores de ingreso y producción hasta lograr impactos positivos en los efectos de la desigualdad, promoviendo a través de un eje transversal la lucha contra la pobreza, la generación de ingresos y el desarrollo de microempresas en países menos desarrollados. Chibba (2009) describe los canales que conectan la inclusión financiera y el desarrollo financiero con indicadores como el coeficiente de Gini; ya que se obtiene una disminución de la pobreza y mayor equidad social en los países que se encuentran en etapas intermedias y avanzadas de uso eficiente de los productos financieros al beneficiar a los sectores más vulnerables.

En este sentido, la inclusión financiera por medio del microcrédito o las microfinanzas surge como una forma de romper el círculo vicioso de la pobreza a través de soluciones y prácticas innovadoras que superan los obstáculos de los problemas de información y reducen los costos de transacción (AMBRÓZIO; GONZALEZ, 2019).

Por otra parte, es importante resaltar que la pobreza rural es la responsable del $63 \%$ de la pobreza mundial; por lo que se constituye como la comunidad de mayor necesidad insatisfecha, así como el más grande mercado desatendido de los servicios financieros; Colombia no es la excepción, y aunque existen grandes avances en temas de reducción de pobreza e inclusión financiera en el país, la población rural sigue siendo la de mayor problemática en este campo (PATIÑO, 2010).

De acuerdo a entidades como Asobancaria y el Banco de la República, la gran mayoría de las personas que viven en poblaciones urbanas tienen acceso a entidades que prestan servicios financieros, pero se excluye en gran medida a las personas de poblaciones rurales, quienes para acceder a ellos, se ven en la necesidad de recorrer grandes distancias o en su defecto acudir a prestamistas informales; por lo que no se desarrolla una cultura financiera de pago y ahorro, lo que dificulta la profundización financiera en este sector. 


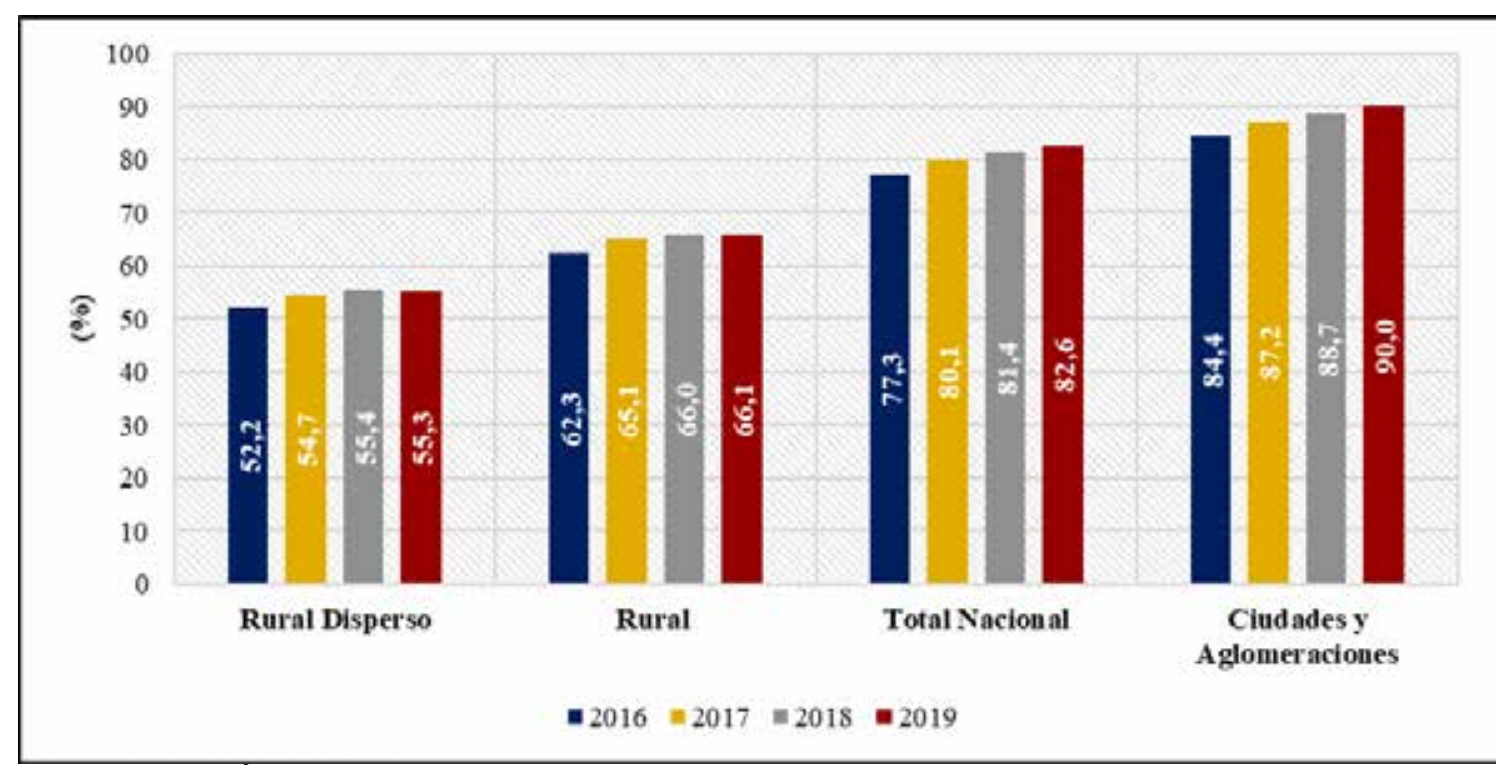

Fuente: Banca de las Oportunidades y Asobancaria.

Las informaciones del Gráfico 1 muestran la capacidad y el espacio de crecimiento que tienen las cooperativas de ahorro y crédito para agregar e incluir a la población rural de Colombia en el sistema financiero nacional, tanto en las áreas rurales convencionales como en las dispersas. A pesar del crecimiento del $3 \%$ al $4 \%$ en el nivel de acceso a productos financieros, todavía existe un espacio de crecimiento muy significativo en comparación con las ciudades y aglomeraciones. Además, las cooperativas de crédito son más que el acceso a productos y servicios financieros, ya que reflejan la inclusión bancaria y financiera. Por un lado, esta inclusión fortalecerá el acceso al crédito, a los servicios bancarios y estimulará la cultura del ahorro y la inversión de recursos. Por otro lado, las poblaciones atendidas por las cooperativas de crédito también se incluyen en los siete pilares del cooperativismo, ya mencionados en este texto. Esto además de ser significativo para la inclusión bancaria, también los es para la inserción en los principios de educación financiera, la gestión democrática y la equidad entre los miembros, lo cual fortalece los lazos de cooperación y de las acciones colectivas.

Gardeva y Rhyne (2011) señalan la importancia de la educación financiera ya que debe ser el complemento del acceso a los productos financieros, pues uno de los motivos por los que la población rural no demanda productos ni servicios financieros es porque no tienen conocimiento de su existencia, ni de su manejo. Además, aquellas entidades que deberían ofrecerles estos productos y servicios no conocen las necesidades verdaderas de la población rural porque no se trata solamente de investigación de mercado, sino de una investigación integral que facilite el entendimiento en el contexto local de las necesidades, expectativas y tendencias de la población rural.

Figueiredo et al. (2018) considera que el acceso al crédito y financiamiento son importantes para el fortalecimiento de la agricultura, así como para el dinamismo y el crecimiento económico de las regiones.

En este mismo pensamiento, Fraga, Ferreira y Teodósio (2020) entienden que las microfinanzas y sus diversos instrumentos, como el microseguro y el microcrédito, priorizan las buenas 
relaciones entre las partes. Según estos autores, la promoción del acceso al crédito también debe entenderse como un transformador de cambio esencial para el desarrollo sostenible, integrado con el objetivo de superar la pobreza.

En la literatura se puede encontrar diferentes causas a la baja accesibilidad de productos financieros principalmente en sectores rurales; según Beck y De la Torre (2007), la naturaleza de las barreras suele ser geográficas (zonas rurales remotas y dispersas), socioeconómicas (costos altos, discriminación de género, étnica, etc.) y de oportunidades (falta de garantías o de pertenencia a una red bien conectada). Por su parte, Honohan (2004), distingue entre barreras de precio (servicios financieros demasiado caros), barreras de información (asimetrías de información en la concesión de créditos que impiden la realización de contratos), y barreras de productos y servicios (servicios que a pesar de ser muy demandados por ciertos grupos no son ofrecidos).

Son muchos los ejemplos internacionales y nacionales que se podrían nombrar casos de éxito en inclusión financiera rural; como ejemplo, puede mencionarse al Grameen Bank, creado por el ganador del premio nobel de la paz, Muhammad Yunus, quien preocupado por la pobreza Bangladesh, fundó un sistema bancario que presta pequeños montos de dinero a pobres en áreas rurales, en especial a mujeres, los cuales son utilizados para la creación de microempresas.

Otro caso de éxito lo sitúan Karlan y Zinman (2010) en Sudáfrica con miras a la ampliación del acceso al crédito de consumo donde se observó un incremento del bienestar de los prestatarios: los ingresos y el consumo de alimentos aumentaron, los indicadores relativos a la toma de decisiones en el hogar mejoraron, la situación de los prestatarios en la comunidad mejoró al igual que la salud en general y el panorama en materia de perspectivas y posición. Sin embargo, los prestatarios también estaban sujetos en mayor grado a situaciones de estrés.

Echavarría et al. (2017) utilizaron los microdatos del Censo Nacional Agropecuario del 2014 para evaluar el impacto del otorgamiento de crédito en el sector agropecuario colombiano y comprobaron que el otorgamiento de crédito reduce el índice de pobreza multidimensional en una magnitud de 0,3 puntos porcentuales.

Finalmente, Rodríguez Garcés (2008) se encarga de evidenciar la relación directa entre el crédito y el empoderamiento de las mujeres beneficiarias, que es tan mencionado por los defensores del impacto del microcrédito, como uno de los efectos positivos del mismo dependiendo de la cultura, pues hay comunidades donde los esposos y los hijos toman las decisiones sobre el uso del préstamo a pesar de que sea la mujer la receptora de este. El impacto también depende de los fines a los que están orientadas las organizaciones que operan los servicios micro-financieros, pues algunas están mucho más enfocadas en promover el desarrollo empresarial y micro-financiero, que otras.

\section{METODOLOGÍA}

Una vez definido el contexto y la problemática de la inclusión financiera, principalmente en el sector rural se procede a realizar un estudio de caso para analizar los usuarios de una entidad financiera colombiana que hace parte de un universo poblacional; con el fin de reseñar rasgos, cualidades o atributos de los agricultores e individuos que residan en el sector rural. Se parte de la hipótesis que los agricultores tienen un buen hábito de pago de sus obligaciones 
financieras y se evaluará la voluntad de pago respecto los demás perfiles que cuenta la entidad financiera en particular.

La fuente de información son datos secundarios proporcionados por la entidad financiera del sector real, objeto de estudio, para el periodo comprendido entre el año 2018 y 2019, que serán anonimizados para ocultar la identificación de individuos particulares pues la información relevante corresponde a los microdatos de las variables sociodemográficas y se expondrá únicamente los resultados del modelo desarrollado, reduciendo los riesgos asociados al tratamiento masivo de datos personales.

La muestra correspondiente a la ventana de tiempo de 2 años seleccionados permite trabajar con un total de 43.295 clientes que cuentan con créditos desembolsados. Inicialmente se elaborará un análisis descriptivo de los créditos otorgados por la entidad financiera objeto de estudio entre los años 2018-2019 y determinar las principales características sociodemográficas de ellos.

Posteriormente se identificarán las zonas geográficas del país más riesgosas de acuerdo con el incumplimiento/impago de los clientes en sus obligaciones financieras y se analizará la actividad principal de dichos departamentos para tratar de validar la correlación con la voluntad de pago de los agricultores. El análisis descriptivo de la muestra y la participación por departamentos se realizará a través del software estadístico Tableau 10.5.

Finalmente, se pretende concluir con los principales beneficios que otorga la inclusión financiera a las comunidades vulnerables, especialmente a los agricultores y las debilidades y oportunidades que se presentan en este campo de cara al corto, mediano y largo plazo, teniendo en cuenta que el Programa de las Naciones Unidas para el Desarrollo (PNUD) tiene como uno de sus pilares la erradicación de la pobreza extrema y el hambre, por lo que el apoyo al sector rural a través del acceso al crédito es indispensable para mejorar la productividad y los ingresos en este sector.

\section{RESULTADOS Y DISCUSIONES}

A continuación, se presenta un análisis descriptivo de la muestra de 43.295 individuos que cuentan con créditos desembolsados y se encuentran en las bases de datos de la entidad financiera objeto de estudio.

El Gráfico 2 presenta el porcentaje de impago por variables sociodemográficas, como se puede observar, se encuentra que las ocupaciones principales reportadas por los clientes son: prestadores de servicio o contratistas que representaron el 31\% (13. 421 personas) de la muestra y se calcula una probabilidad de impago promedio del $20 \%$ frente a las demás ocupaciones, seguido por los agricultores que representan el 10\% (4.330 personas) de la muestra y muestran una probabilidad de impago del $18 \%$. Los ocupados en la modalidad de empleados tienen una alta participación, al representar el $43 \%$ del total (18.677 personas) y un índice de impago del $13 \%$.

En general, las actividades de los prestadores de servicios o contratistas son las que presentan mayores fluctuaciones en los negocios y son más frágiles ante los ciclos económicos. Cuando la economía no se encuentra en una situación sostenible, los consumidores tienden a cancelar las actividades de prestación de servicios en primer lugar, lo que hace que esta activi- 
dad se vea más afectada tanto en las crisis como en la capacidad de pago. De la misma forma, la mejora de las condiciones de vida de la población tiene un impacto directo en el aumento de la contratación de servicios. Los agricultores se encuentran en una situación diferente. Incluso con precios bajos y condiciones de mercado complicadas, tienden a sembrar y cuidar su estructura productiva, lo que les garantiza un volumen de ingresos en los ciclos agrícolas. Solo problemas climáticos o algún endeudamiento en vista de su capacidad de ingresos son factores que pueden afectar su capacidad de pago.

Gráfico 2 - Impago, participación y cantidad de clientes por variable sociodemográfica

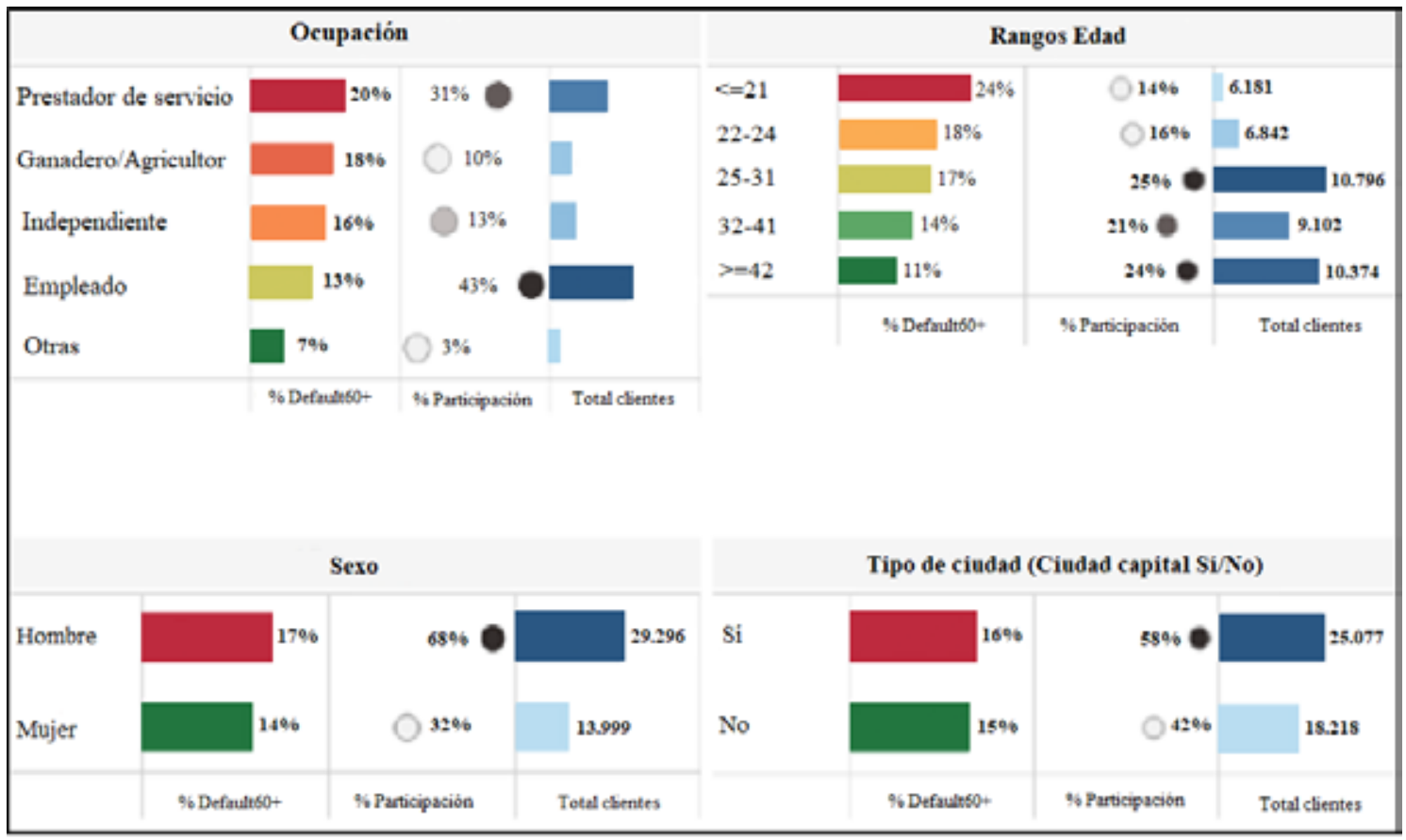

Fuente: Elaboración propia con datos de la entidad financiera.

Respecto a los rangos de edad se puede observar que la tasa de impago cae a medida que aumenta la edad de las personas. De esta manera, los clientes que no superan los 21 años representan el $14 \%$ del total y registran un impago de $24 \%$, comparado con los clientes con edades superiores a los 41 años que alcanzan un impago de $11 \%$ y representan el $24 \%$ de la muestra.

Para la variable Sexo se obtiene que la mayoría de los créditos otorgados están dirigidos a hombres con un $68 \%$ mientras que las mujeres representan el $32 \%$ restante y resalta que la tasa de impago es similar en ambos casos.

En el caso del acceso al crédito, en general, la estructura patriarcal del "hombre proveedor" se refleja mucho en la toma de crédito. Sin embargo, existe un mayor compromiso de las mujeres para honrar las deudas y mantener su acceso al crédito. En este sentido, los datos llevan a reflexionar sobre las carteras y las políticas crediticias más específicas para las mujeres, en particular fomentando el emprendimiento y el empoderamiento femenino.

A su vez, se observa que $58 \%$ de los créditos fueron desembolsados en ciudades capitales (25.077 clientes). Las tasas de impago son similares para las personas según lugar de residen- 
cia. Al respecto, los clientes en las ciudades capitales tienen un default de $16 \%$ mientras que aquellos que viven en ciudades no capitales se ubican en $15 \%$.

Con base en las estadísticas descriptivas para la entidad financiera objeto de estudio, los perfiles menos atractivos para incentivar el acceso al crédito son los prestadores de servicio o contratistas y los agricultores dadas las tasas de impago, $20 \%$ y $18 \%$ respectivamente. Sin embargo, es importante resaltar que las políticas internas de colocación para estos perfiles son las más flexibles, pues no requieren soportes para estudio crediticio muy elaborados, ni se puede comprobar con certeza el desempeño en sus actividades; por lo que probablemente se filtren diferentes perfiles bajo la sombra de contratistas o agricultores.

Estas informaciones reflejan la fragilidad de las actividades primarias y terciarias en Colombia. Asimismo, los datos del Gráfico 2 refuerzan la necesidad de líneas de crédito creadas a través de políticas públicas para apoyar las actividades más frágiles de la economía colombiana. Buena parte del impago se debe a la fragilidad del negocio y en menor proporción a la mala voluntad de los deudores. Por esta razón, las cooperativas de ahorro y crédito pueden ser un instrumento de líneas de financiamiento subsidiadas y especiales creadas por el Banco Central de Colombia o por organismos gubernamentales para fortalecer el empleo, los ingresos y las condiciones de vida de los agricultores, micro y pequeños empresarios. Un ejemplo de ello es el microcrédito, con plazos largos, tanto de pago como de gracia, y a tipos de interés cero o especiales.

Se debe tener en cuenta que los jóvenes que habitan en el sector rural (entre 18 y 25 años) son los que cuentan con una menor tasa de ingreso al sistema financiero, con un indicador de acceso de alrededor del 30\% (ASOBANCARIA, 2019). Sin embargo, las entidades financieras al registrar tasas de impago cada vez mayores entre menor sea la edad de los individuos, difícilmente se encargarán de promover políticas de inclusión financiera hacia los más jóvenes; por lo que es importante que se desarrollen políticas de educación financiera en este tipo de comunidades que concienticen a los jóvenes de los beneficios y el impacto que puede generar en la calidad de vida.

Cabe recordar que las acciones de educación financiera cobraron cada vez más importancia después de la crisis financiera de 2008. Con esta crisis, la situación de la capacidad de pago de los jóvenes en varias partes del mundo se vio comprometida, debido al aumento del desempleo, que generó la dificultad para administrar sus ingresos y sus pagos (RIPPEL; RIPPEL, 2008). Esto demuestra que las cooperativas de ahorro y crédito también pueden actuar para fortalecer la inserción de los jóvenes y crear mecanismos para reducir el impacto de las crisis, tanto en las acciones de acceso al crédito como en el uso racional del crédito.

En cuanto a la variable sexo se evidencia en la colocación de los créditos que los hombres duplican los desembolsos de créditos a las mujeres; lo que puede reflejar la sociedad latinoamericana donde persiste el paradigma que el hombre es quien dirige el rumbo de los hogares $y$ dispone de los recursos económicos. Aún se encuentra mucho camino por recorrer para empoderar a las mujeres a través del acceso a la financiación de proyectos y emprendimientos que generen progreso social.

El tipo de ciudad en la que se encuentran los clientes proyecta una dispersión en la colocación en la mayoría del territorio nacional y no solo en las ciudades principales, los municipios, cabeceras y sector rural representan un considerable $42 \%$ de los desembolsos crediticios; 
demostrando avances en materia de penetración de mercado e inclusión en las ciudades no capitales y contrarrestando las actividades de prestamistas informales que socavan los intereses particulares de las comunidades más vulnerables.

En un estudio hecho por Ramborger et al. (2019) se notó que los cambios en el ambiente externo, en el crecimiento de las cooperativas y en la heterogeneidad de los cooperativistas aumentan los riesgos de las cooperativas que brindan o dependen del crédito agrícola. Es por esto que los modelos de gestión de las cooperativas deben ser técnicos, a pesar de su necesidad de actuar también con una fuerte responsabilidad social. Los datos de las carteras crediticias de Colombia refuerzan esta percepción.

El Gráfico 3 presenta el porcentaje de impago por departamentos, la cantidad de clientes y la participación correspondiente. Se puede observar Respecto al índice de impago se presentan diferencias notorias a nivel departamental. Los departamentos de Cesar, Sucre y Córdoba registran las mayores tasas de impago, $32 \%, 25 \%$ y $22 \%$, respectivamente. La ciudad capital (Bogotá), aglomera el 15\% de la muestra con 6.449 clientes y presenta un impago (default) de $16 \%$. Por otra parte, Antioquia, Quindío y Risaralda tienen las más bajas tasas de impago que no superan el $12 \%$. En cuanto al Valle del Cauca, donde reside el $21 \%$ de la población, tiene un índice de default de $12 \%$.

Gráfico 3 - Impago, cantidad de clientes y participación por departamentos

\begin{tabular}{|c|c|c|c|c|}
\hline \multicolumn{4}{|c|}{ Departamentos } & Mapa Impagos por Departamentos \\
\hline CESAR & $32 \%$ & $2 \%$ & 1028 & \\
\hline SUCRE & $25 \%$ & $1 \%$ & 1547 & pert: \\
\hline CÓRDOBA & $22 \%$ & $4 \%$ & 1.559 & \\
\hline BOLÍVAR & $21 \% 0$ & $30 \%$ & 1.460 & \\
\hline N. DE SANTANDER & $20 \%$ & $3 \%$ & 1.121 & \\
\hline SANTANDER & $19 \%$ & $5 \%$ & 2036 & \\
\hline TOLIMA & $19 \%$ & $7 \%$ & 3.167 & \\
\hline ATLÁNTICO & $19 \%$ & $2 \%$ & 920 & \\
\hline MAGDALENA & $18 \%$ & $1 \%$ & 5.44 & \\
\hline CAQUETÁ & $18 \%$ & $4 \%$ & 1.791 & Irow \\
\hline $\begin{array}{l}\text { BOGOTÁ } \\
\text { CALDAS }\end{array}$ & $\begin{array}{l}16 \% \bigcirc \\
16 \%\end{array}$ & $15 \%$ & \begin{tabular}{l|l}
5.419 \\
540
\end{tabular} & Mapa Calor Impago Departamentos \\
\hline HUILA & $15 \%$ & $3 \%$ & 1.165 & \\
\hline CUNDINAMARCA & $15 \%$ & $5 \%$ & 2299 & nte. \\
\hline META & $14 \%$ & $10 \%$ & 234 & \\
\hline NARIÑo & $13 \% \bigcirc$ & $5 \%$ & 2.014 & \\
\hline CAUCA & $13 \% \%$ & $11 \%$ & 4.565 & \\
\hline VALLE DEL CAUCA & $12 \%$ & $21 \%$ & 9.059 & \\
\hline ANTIOQUIA & $11 \% 0$ & $30 \%$ & 1310 & \\
\hline QUINDío & $8 \%$ & $1 \%$ & 408 & \\
\hline \multirow[t]{2}{*}{ RISARALDA } & $70 \%$ & $2 \%$ & 749 & \\
\hline & $9 \%$ Default $60+$ & $\%$ Participación & Total clientes & INSW \\
\hline
\end{tabular}

Fuente: Elaboración propia con datos de la entidad financiera.

Con el ranking de impago por departamentos se puede observar que departamentos agrícolas como: Quindío (Café, plátano, cacao), Valle del Cauca (Caña de azúcar, banano, aguacate), Cauca (Fique, Caña de azúcar, café), Nariño (Papa, cacao, maíz) y Meta (Arroz, palma africana, plátano) reflejan bajas tasas de impago crediticio mientras que departamentos donde la gana- 
dería ocupa un lugar representativo en su actividad económica como: Cesar, Sucre, Córdoba y Bolívar presentan las tasas más altas de impago.

Estos datos demuestran el perfil del impago en relación con la posición geográfica de los departamentos. En la parte norte del territorio colombiano y en la zona amazónica los impagos son mayores. Esto también se relaciona con la participación de estas regiones en la economía colombiana. Información de la Confederación de Cooperativas de Colombia (Confecoop) en la que se señala que las regiones donde se concentra el mayor porcentaje de cooperativas, tanto en número de socios, como en entidades y volumen de activos, es el Eje Cafetero y Antioquia, con el $50 \%$ y $63 \%$ de la participación total nacional, respectivamente. La región de Antioquia concentra el $13 \%$ del PIB nacional.

Cabe recordar que el ingreso rural equivale al $31 \%$ del ingreso urbano en Colombia, es decir, la capacidad de pago de las poblaciones rurales y campesinas es más frágil si su ocupación es en actividades rurales, en comparación con las actividades urbanas. Sin embargo, al analizar los datos de los gráficos 1, 2 y 3, se observa que aún existe un riesgo menor en los préstamos de las actividades rurales comparados con los de las actividades urbanas. Y las actividades urbanas con mayor riesgo de impago se ubican en los departamentos con menor dinamismo económico. Es decir, en Colombia las condiciones de pago están diferenciados espacialmente, tanto geográfica como económicamente.

Un ejemplo del uso de las cooperativas de ahorro y crédito como instrumento de desarrollo rural y agroindustrial es el caso de Brasil. En dicho país, se hizo evidente que las cooperativas eran un instrumento importante para el desarrollo económico rural y urbano. Además de su inserción en el interior del país, donde la banca convencional no tenía interés en operar, las cooperativas fueron intermediarias de créditos públicos para la modernización de las zonas rurales, estimulando la comercialización garantizando el financiamiento de los costos y el transporte de los cultivos. Actualmente, su importancia es vista como estratégica, no solo para el desarrollo socioeconómico, sino también para evitar la concentración financiera en la banca comercial, lo que refuerza la historia de las cooperativas que surgieron como oposición a los monopolios privados (ALVES; FERRERA DE LIMA, 2018).

\section{CONCLUSIONES}

A partir de la inclusión financiera y el acceso al crédito se permitirá a la población de microempresarios en zonas rurales y a los pequeños y medianos productores desarrollar emprendimientos, mejorar los ingresos, la calidad de vida y acceder a otros servicios como los seguros y la educación financiera.

Las condiciones de los créditos de entidades financieras formales son sustancialmente mejores que las alternativas de crédito informal tanto en tasa como en plazo. Los gota a gota o prestamistas informales representan un problema muy complejo en el marco del desarrollo personal y colectivo de las comunidades vulnerables y terminan siendo un generador de violencia y pobreza, generando un círculo vicioso del que difícilmente se pueden desprender quienes acceden a estas formas de financiación.

Las mujeres están en una situación de especial desventaja en cuanto al acceso a servicios financieros, tienen menos acceso a mecanismos seguros de ahorro y más probabilidades de 
usar métodos informales, por lo que es indispensable promover políticas públicas y privadas que logren equilibrar las oportunidades de acceso a productos financieros.

La inclusión financiera importa no solo porque promueve el crecimiento, sino que ayuda además a garantizar que la prosperidad sea ampliamente compartida, disminuyendo los índices de desigualdad social. El acceso a los servicios financieros juega un papel fundamental en la tarea de sacar a la gente de la pobreza, empoderar a las mujeres y ayudar a los gobiernos a prestar diferentes servicios a la población. Es una herramienta decisiva en la lucha contra la pobreza.

La gran heterogeneidad social en el contexto nacional sugiere que las políticas de inclusión financiera deben ir encaminadas a fomentar el acceso de la población al sistema financiero deben ir focalizadas a grupos específicos, tanto a nivel de edad como en ubicación geográfica. En particular, los jóvenes adultos y los habitantes del sector rural son los que requieren una atención más detallada por parte de estas iniciativas. Para lograr tal fin, es necesario fortalecer los programas de educación financiera actuales.

Las estadísticas encontradas no permiten confirmar el buen hábito de pago del perfil de los agricultores, se evidencia que inciden otras variables de manera más significativa como la ubicación geográfica y la edad.

Mejorar las condiciones de vida de las poblaciones vulnerables dependen del acceso a una amplia gama de recursos donde se encuentra el acceso a la financiación y la posibilidad de ahorro. Es vital el relacionamiento de actores estatales, de mercado y sociedad civil para la superación de la pobreza a través de la integración de fuerzas y diferentes recursos.

Entender las causas y el contexto de los contrastes sociales presentes en Colombia resulta ser un elemento crucial que debe tenerse en cuenta para el diseño de políticas públicas y privadas que promuevan la inclusión financiera. El análisis sugiere que el grado de acceso a servicios financieros a nivel local no solamente está asociado a condiciones económicas locales sino también a otros elementos sociales. Por lo que, el éxito de una política de desarrollo financiero en Colombia depende, entre otros factores, de la capacidad de establecer tanto condiciones financieras como socioeconómicas propicias a nivel local.

En este contexto, se entiende que brindar a los territorios rurales colombianos servicios financieros bien estructurados y capaces de atender la demanda crediticia de la porción de la población excluida del sistema bancario representa una acción de primera necesidad cuando se desea promover el desarrollo de una región.

Es difícil concluir con los datos proporcionados por la entidad financiera objeto de estudio el desempeño crediticio de los agricultores colombianos, dado la baja representatividad de la muestra y las estadísticas variadas que se encontraron en el análisis. Más que la actividad propia de los solicitantes de crédito se debe tener en cuenta la edad y la cultura de pago desarrollada en las distintas zonas geográficas ya que se puede remarcar una cultura responsable y cumplidora hacia el suroccidente colombiano y una cultura de muy mal hábito de pago sobre la costa atlántica.

Es importante recordar que este estudio demostró que en Colombia las condiciones de pago, el acceso al crédito y la inclusión financiera están diferenciadas geográfica y económicamente. En las regiones más frágiles y con escasa participación en la economía nacional, las condiciones de impago y acceso al crédito son más débiles. Esto significa que el acceso al crédi- 
to también debe ser visto como un elemento de política pública para estimular las economías regionales y promover el desarrollo regional.

Para próximos estudios referentes al tema de la inclusión financiera en el sector rural y el desempeño crediticio de los agricultores es conveniente realizar estudios etnográficos que permitan experimentar y conocer de primera mano el contexto y las condiciones de este tipo de comunidades, pues el análisis de datos por si solo generaliza y reafirma paradigmas que no favorecen a las comunidades vulnerables y por lo tanto no contribuyen a la mitigación de la pobreza.

\section{REFERENCIAS}

ALVES, L.; FERRERA DE LIMA, J. Cooperativismo agropecuário e de crédito no Brasil do agronegócio. Revista Brasileira de Gestão e Desenvolvimento Regional, v. 14, n. 2, p. 558-587, 2018.

AMBRÓZIO, L.; GONZALEZ, L. O Programa Minha Casa Minha Vida e as tecnologias de microcrédito: contribuições para uma agenda de pesquisa. Desenvolvimento em Questão, v. 17, n. 47, p. 120-135, 2019. DOI: 10.21527/2237-6453.2019.47.120-135

ASOBANCARIA. Asociación Bancaria y de Entidades Financieras de Colombia. Estrategia de inclusión financiera en Colombia 2019-2022. Revista Semana Económica. Edición 1.206, 2019.

ASOBANCARIA. Asociación Bancaria y de Entidades Financieras de Colombia. Estudios en material de inclusión financiera en 2017: principales resultados y reflexiones para Colombia. Revista Semana Económica, Edición 1.153, 2018.

BECK, T.; DE LA TORRE, A. The Basic Analytics of Access to Financial Services. Financial Markets, Institutions \& Instruments, 16(2), p. 79-117, 2007.

CHIBBA, M. Financial Inclusion, Poverty Reduction and the Millennium Development Goals. European Journal of Development Research, n. 21, p. 213-230, 2009.

DAMBROS, M.; FERRERA DE LIMA, J.; FIGUEIREDO, A.M. Sistema cooperativo de crédito Sicredi: um estudo da eficiência das cooperativas do Paraná. Gestão \& Regionalidade, 25 (74), p. 22-34, 2009. DOI: 10.13037/gr. vol25n74.141.

DANE. Departamento Administrativo Nacional de Estadística. Medición de empleo informal y seguridad social. Boletín Técnico Trimestre móvil, jun./ago. 2019a.

DANE. Departamento Administrativo Nacional de Estadística. Principales indicadores del mercado laboral. Boletín Técnico, sept. 2019b.

DANE. Departamento Administrativo Nacional de Estadística. Boletín Técnico - Pobreza Monetaria en Colombia, 2018. Disponível em: https://www.dane.gov.co/

DNP. Departamento Nacional de Población. Definición de Categorías de Ruralidad, 2014. Disponível em: https://colaboracion.dnp.gov.co/

ECHAVARRÍA, J. J.; VILLAMIZAR-VILLEGAS, M.; RESTREPO-TAMAYO, S.; HERNÁNDEZ-LEAL, J. D. Impacto del crédito sobre el agro en Colombia: evidencia del nuevo censo nacional agropecuario. Banco Interamericano de Desarrollo, 2017.

FEDESARROLLO. Informe Mensual del Mercado Laboral. Productividad y Salario Mínimo, 2018. Disponível em: https://www.repository.fedesarrollo.org.co/bitstream/handle/11445/3527/IML_Enero_2018. pdf?sequence=1\&isAllowed=y

FIGUEIREDO, A. K. S. de; PIRES, M. de M.; GOMES, A. da S.; MOROLLON, F. R. Análise espacial do desenvolvimento e das desigualdades no território sudoeste baiano. Desenvolvimento em Questão, v. 16, n. 44, p. 69-104, 2018. DOI: 10.21527/2237-6453.2018.44.69-104

FRAGA, M. S.; FERREIRA, C. A. A.; TEODÓSIO, A. dos S. de S. Elementos emancipatórios na pobreza multidimensional: a gestão dos ativos intangíveis e do capital social. Desenvolvimento em Questão, v. 18, n. 53, p. 183-201, 2020. DOI: 10.21527/2237-6453.2020.53.183-201

GARDEVA, A.; RHYNE, E. Oportunidades y obstáculos para la inclusión financiera. Informe de la encuesta. Centro para la Inclusión Financiera, 2011.

HILFERDING, R. O capital financiero. São Paulo: Nova Cultural, 1985.

HONOHAN, P. Financial Sector Policy and the Poor: Selected Findings and Issues. The World Bank Working Paper, Washington D. C., n. 43, 2004. 
KARLAN, D.; ZINMAN, J. Expanding credit access: Using randomized supply decisions to estimate the impacts. Review of Financial Studies, v. 23, n. 1, p. 433-464, 2010. DOI 10.1093/rfs/hhp092.

MORDUCH, J. Income Smoothing and Consumption Smoothing. Journal of Economic Perspectives, v. 9, n. 3, p. 103-114, 1995.

OCDE; INFE (Organización de Cooperación y Desarrollo Económicos/Red Internacional de Educación Financiera). "Principios de Alto Nivel de la OCDE/INFE sobre Estrategias Nacionales de Educación Financiera", 2012. Disponível em: https://www.oecd.org/finance/nationalstrategiesforfinancialeducation.htm. PATIÑO-CASTRO, O. A. (2010). El modelo MIFE como instrumento para la formalización del empresariado. Una aplicación para las microempresas informales de Bogotá D.C. 287 pgs. Tese de Doutorado, Universidad Nebrija, Madrid. DOI 10.21158/9789587561081.

RAMBORGER, B. M.; BORBA, M. C.; KINDLEIN, L.; MACHADO, J. A. Ambientes competitivos dinâmicos e tomada de decisões ágil: competências para a sustentabilidade organizacional cooperativista. Informe Gepec, v. 23, n. 2, p. 156-169, 2019. DOI: 10.48075/igepec.v23i2.22572

RIPPEL, R.; RIPPEL, V. L. Considerações a respeito das origens, dos acontecimentos e das consequências da crise econômica mundial atual. Informe Gepec, v. 12, n. 2, p. 6- 27, 2008. DOI: 10.48075/igepec. v12i2.2298

RODRÍGUEZ GARCÉS, C. Impacto de las microfinanzas: resultados de algunos estudios. Énfasis en el sector financiero. Revista Ciencias Estratégicas, v. 16, n. 20, p. 281-298, 2008.

SCHUMPETER, J. A. Teoria do desenvolvimento econômico: uma investigação sobre lucros, capital, crédito e juros e o ciclo econômico. São Paulo: Abril Cultural, 1982. 